\title{
无溶剂无催化剂条件下二硫代氨基甲酸酯衍生物的合成研究
}

\author{
郭圣荣*袁艳琴张春牛 \\ (丽水学院化学化工系 丽水 323000)
}

\begin{abstract}
摘要 主要研究了二硫代氨基甲酸酯衍生物的合成, 将脂肪胺、 $\mathrm{CS}_{2}$ 与 $\alpha, \beta$-不饱和羰基化合物或卤代烃在无溶剂无催化 剂室温条件下，“一锅法” 进行了 Michael-type 的加成反应，合成了二硫代氨基甲酸酯衍生物。该方法产率高，操作简 单，是一种原子经济的合成方法，并可大规模地用于制药及农药方面.

关键词 二硫代氨基甲酸酯; 无溶剂; 二硫化碳; $\alpha, \beta$-不饱和羰基化合物
\end{abstract}

\section{Highly Efficient Catalyst-Free One-Pot Synthesis of Dithiocarbamates under Solvent-Free Conditions}

\author{
Guo, Shengrong* Yuan, Yanqin Zhang, Chunniu \\ (Department of Chemistry, Lishui University, Lishui 323000)
}

\begin{abstract}
A highly efficient and simple synthesis of dithiocarbamates is reported in the one-pot reaction of amines, $\mathrm{CS}_{2}$, and alkyl halides without using a catalyst under solvent-free conditions. The mild reaction conditions, high yields, and broad scope of the reaction illustrate the good synthetic utility of this method. The reaction is a highly atom-economic process for production of dithiocarbamates and can be successfully used in large amount for the pharmaceutical or agrochemical industries.

Keywords dithiocarbamates; solvent-free; carbon disulfide; $\alpha, \beta$-unsaturated compounds
\end{abstract}

二硫代氨基甲酸酯衍生物是一类应用广泛的化合 物, 具有较好的生理活性 ${ }^{[1 \sim 3]}$ 及生物活性 ${ }^{[4 \sim 6]}$, 同时还可 用作硫化剂 $\left[{ }^{[7]}\right.$ 、光引发剂 ${ }^{[8]}$ 等. 该类化合物常用的合成方 法是：在强碱性条件下，伯胺或仲胺与二硫化碳反应生 成对应的氨基二硫代甲酸盐, 再与卤代烷反应, 生成氨 基二硫代甲酸酯, 但由于需要在强碱性条件反应，使反 应底物中不能含有对碱敏感的基团，同时存在中间体需 要分离, 反应时间较长等缺点 ${ }^{[9]}$.

近年来, 有关二硫代氨基甲酸酯合成的文献报道 中, 有两个方法较为突出, 一个是 Saidi 等 ${ }^{[10]}$ 以水为溶 剂及催化剂; 另一个是 Ranu 等 ${ }^{[1]}$ 用离子液体为溶剂及 催化剂, 两者都较为成功地合成了二硫代氨基甲酸酯衍 生物, 而且所用的溶剂都为绿色溶剂, 是绿色有机合成 的较好范例. 但我们发现用水作溶剂时, 反应时间较长, 产物要有大量溶剂萃取, 用离子液体为溶剂时, 虽反应 时间较短, 但产物的分离也需要大量的溶剂来萃取, 实 质上并不是完全意义上的绿色合成.
无溶剂有机合成方法由于具有绿色、经济等特点而 备受关注 ${ }^{[12,13]}$, 是有机合成的热点领域之一. 相对于传 统的溶液反应，它还具有高效性、高选择性、易于分离 纯化产物及反应条件温和等优点, 在药物合成领域中应 用较为广泛 ${ }^{[14]}$. 本文在 Saidi 及 Ranu 方法基础上, 研究 了在无溶剂条件下 “一锅法” 合成二硫代氨基甲酸酯的 反应，实验发现在无溶剂及无催化剂的条件下，反应同 样能较快地完成, 且产率较高, 是一种合成二硫代氨基 甲酸酯较好的方法(Scheme 1).

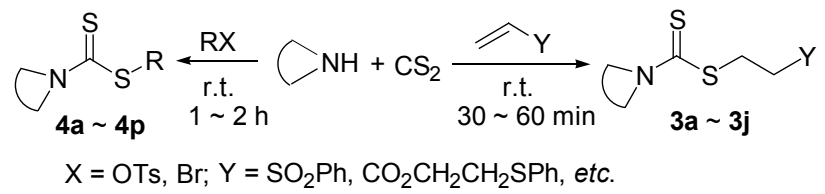

Scheme 1

\footnotetext{
*E-mail: guosr9609@163.com

Received August 17, 2011; revised December 27, 2011; accepted January 10, 2012.

Project supported by the Natural Science Foundation of Zhejiang Province (No. Y407240) and the Key Science Foundation of Lishui University (No. KZ201103).

浙江省自然科学基金(No. Y407240)和丽水学院重点科研(No. KZ201103)资助项目.
} 


\section{1 实验部分}

\section{1 仪器和试剂}

熔点测定仪: Yanagimoto MFG., 温度计未校正; 核 磁共振仪: Bruker Avance 300, 以 TMS 为内标, 氛代氯 仿为溶剂; 元素分析仪: Perkin-Elmer 240C. 所用的药 品均为市售的分析纯或化学纯, 除特别注明外, 未经进 一步处理.

\section{2 合成实验}

在氮气保护下，向 Schlenk 管中加入 $\mathrm{CS}_{2}(6 \mathrm{mmol})$, $\alpha, \beta$-不饱和化合物或卤代烃 $(4 \mathrm{mmol})$, 冰浴冷却到 $0{ }^{\circ} \mathrm{C}$, 磁力搅拌下, 缓缓注入脂肪胺 $(4.5 \mathrm{mmol})$, 冰浴下搅拌 $30 \mathrm{~min}$ 后, 室温下搅拌 $30 \mathrm{~min} \sim 2 \mathrm{~h}$, TLC 跟踪反应, 直 至反应完成. 如反应混合物变为固态, 加入适量的无水 乙醇重结晶就可以得到纯净的产物, 无需过柱纯化; 如 反应混合物认为液态, 直接用过柱分离 $[V$ (乙酸乙酯) : $V($ 环已烷 $)=1: 5$ ]得到目标产物 $\mathbf{3 a} \sim \mathbf{3} \mathbf{j}$ 和 $\mathbf{4 a} \sim \mathbf{4} \mathbf{p}$.

\section{3 产物光谱数据}

1-吡咯烷二硫代氨基甲酸-(2-苯砜基)乙基酯(3a): 白色固体, m.p. $118 \sim 120{ }^{\circ} \mathrm{C}^{[10]}$. ${ }^{1} \mathrm{H}$ NMR $\left(\mathrm{CDCl}_{3}, 300\right.$ MHz) $\delta: 7.92(\mathrm{~d}, J=7.2 \mathrm{~Hz}, 2 \mathrm{H}), 7.56 \sim 7.69(\mathrm{~m}, 3 \mathrm{H}), 3.82$ (t, $J=6.9 \mathrm{~Hz}, 2 \mathrm{H}), 3.47 \sim 3.57(\mathrm{~m}, 6 \mathrm{H}), 1.90 \sim 2.09(\mathrm{~m}$, $4 \mathrm{H}) ;{ }^{13} \mathrm{C}$ NMR $\left(\mathrm{CDCl}_{3}, 75 \mathrm{MHz}\right) \delta: 190.0,138.4,133.9$, $129.3,128.3,55.3,55.0,50.6,28.6,26.0,24.2$. Anal. calcd for $\mathrm{C}_{13} \mathrm{H}_{17} \mathrm{NO}_{2} \mathrm{~S}_{3}$ : C 49.49, H 5.43, N 4.44; found C 49.50, H 5.41, N 4. 46.

1-哌啶烷二硫代氨基甲酸-(2-苯砜基)乙基酯(3b): 白色固体, m.p. $127 \sim 129{ }^{\circ} \mathrm{C}^{[11]}$. ${ }^{1} \mathrm{H}$ NMR $\left(\mathrm{CDCl}_{3}, 300\right.$ MHz) $\delta: 7.95(\mathrm{~d}, J=7.2 \mathrm{~Hz}, 2 \mathrm{H}), 7.55 \sim 7.69(\mathrm{~m}, 3 \mathrm{H}), 4.11$ (br, 2H), 3.77 (br, 2H), 3.53 (t, $J=7.2 \mathrm{~Hz}, 4 \mathrm{H}), 1.65$ (br, $6 \mathrm{H}) ;{ }^{13} \mathrm{C}$ NMR $\left(\mathrm{CDCl}_{3}, 75 \mathrm{MHz}\right) \delta: 193.2,138.7,133.9$, 129.3, 128.3, 55.3, 52.6, 29.1, 25.1, 24.2. Anal. calcd for $\mathrm{C}_{14} \mathrm{H}_{19} \mathrm{NO}_{2} \mathrm{~S}_{3}$ : C 51.03, H 5.81, N 4.25; found C 51.08, H 5.86, N 4. 26.

$N, N$-二乙氨基二硫代甲酸-(2-苯砜基)乙基酯(3c): 白色固体, m.p. 99 101 ${ }^{\circ} \mathrm{C}^{[11]} .{ }^{1} \mathrm{H}$ NMR $\left(\mathrm{CDCl}_{3}, 300\right.$ MHz) $\delta: 7.94(\mathrm{~d}, J=7.2 \mathrm{~Hz}, 2 \mathrm{H}), 7.55 \sim 7.69(\mathrm{~m}, 3 \mathrm{H}), 3.92$ (q, $J=3.2 \mathrm{~Hz}, 2 \mathrm{H}), 3.64(\mathrm{q}, J=4.1 \mathrm{~Hz}, 2 \mathrm{H}), 3.46 \sim 3.58$ (m, 4H), $1.17 \sim 1.24(\mathrm{~m}, 6 \mathrm{H}) ;{ }^{13} \mathrm{C} \mathrm{NMR}\left(\mathrm{CDCl}_{3}, 75 \mathrm{MHz}\right)$ $\delta: 193.2,138.7,133.8,129.3,128.3,55.3,49.6,46.8,29.1$, 12.5, 11.5. Anal. calcd for $\mathrm{C}_{13} \mathrm{H}_{19} \mathrm{NO}_{2} \mathrm{~S}_{3}: \mathrm{C} 49.18, \mathrm{H}$ 6.03, N 4.41; found C 49.14, H 6.06, N 4. 44.

1-丁氨基二硫代甲酸-(2-苯砜基)乙基酯(3d): 白色 固体, m.p. $122 \sim 124{ }^{\circ} \mathrm{C}^{[10]} .{ }^{1} \mathrm{H} \mathrm{NMR}\left(\mathrm{CDCl}_{3}, 300 \mathrm{MHz}\right)$ $\delta: 7.89(\mathrm{~d}, J=7.2 \mathrm{~Hz}, 2 \mathrm{H}), 7.56 \sim 7.65(\mathrm{~m}, 3 \mathrm{H}), 3.55(\mathrm{q}$,
$J=3.6 \mathrm{~Hz}, 2 \mathrm{H}), 3.43(\mathrm{br}, 1 \mathrm{H}), 3.31 \sim 3.50(\mathrm{~m}, 4 \mathrm{H}), 1.50 \sim$ $1.59(\mathrm{~m}, 2 \mathrm{H}), 1.31 \sim 1.35(\mathrm{~m}, 2 \mathrm{H}), 0.87(\mathrm{t}, J=7.1 \mathrm{~Hz}, 2 \mathrm{H})$; ${ }^{13} \mathrm{C}$ NMR $\left(\mathrm{CDCl}_{3}, 75 \mathrm{MHz}\right) \delta: 195.0,138.4,134.0,129.5$, 128.2, 55.6, 47.2, 30.1 27.6, 20.1, 13.7. Anal. calcd for $\mathrm{C}_{13} \mathrm{H}_{19} \mathrm{NO}_{2} \mathrm{~S}_{3}: \mathrm{C} 49.18, \mathrm{H} 6.03, \mathrm{~N} 4.41$, found $\mathrm{C} 49.16, \mathrm{H}$ $6.05, \mathrm{~N} 4.43$

3-(1-吡咯烷二硫代氨基甲酸基)丙酸 (2-苯硫基乙 基)酯(3e)：淡黄色油状液体. ${ }^{1} \mathrm{H} \mathrm{NMR}\left(\mathrm{CDCl}_{3}, 300 \mathrm{MHz}\right)$ $\delta: 7.37 \sim 7.40(\mathrm{~m}, 2 \mathrm{H}), 7.21 \sim 7.38(\mathrm{~m}, 3 \mathrm{H}), 4.28(\mathrm{t}, J=6.9$ $\mathrm{Hz}, 2 \mathrm{H}), 4.26$ (br, 2H), 3.84 (br, 2H), 3.55 (t, $J=6.6 \mathrm{~Hz}$, $2 \mathrm{H}), 3.16$ (t, $J=6.9 \mathrm{~Hz}, 2 \mathrm{H}), 2.79$ (t, $J=6.6 \mathrm{~Hz}, 2 \mathrm{H}), 1.68$ (s, 6H); ${ }^{13} \mathrm{C}$ NMR $\left(\mathrm{CDCl}_{3}, 75 \mathrm{MHz}\right) \delta: 194.7,171.8,135.1$, $129.8,129.1,126.6,63.2,51.3,33.9,32.3,31.5,25.5,24.3$. Anal. calcd for $\mathrm{C}_{17} \mathrm{H}_{23} \mathrm{NO}_{2} \mathrm{~S}_{2}$ : C 60.50, H 6.87, N 4.15; found $\mathrm{C} 60.55, \mathrm{H} 6.84, \mathrm{~N} 4.16$.

3-( $N, N$-二乙氨基二硫代甲酸基)丙酸 (2-苯硫基乙 基)酯(3f)：淡黄色油状液体. ${ }^{1} \mathrm{H} \mathrm{NMR}\left(\mathrm{CDCl}_{3}, 300 \mathrm{MHz}\right)$ $\delta: 7.41(\mathrm{~d}, J=7.2 \mathrm{~Hz}, 2 \mathrm{H}), 7.19 \sim 7.38(\mathrm{~m}, 3 \mathrm{H}), 4.28(\mathrm{t}$, $J=6.9 \mathrm{~Hz}, 2 \mathrm{H}), 4.05$ (q, $J=3.2 \mathrm{~Hz}, 2 \mathrm{H}), 3.72$ (q, $J=3.2$ $\mathrm{Hz}, 2 \mathrm{H}), 3.54$ (t, $J=6.6 \mathrm{~Hz}, 3 \mathrm{H}), 3.15(\mathrm{t}, J=6.9 \mathrm{~Hz}, 2 \mathrm{H})$, $2,78(\mathrm{t}, J=6.6 \mathrm{~Hz}, 2 \mathrm{H}), 1.28(\mathrm{t}, J=6.6 \mathrm{~Hz}, 6 \mathrm{H}) ;{ }^{13} \mathrm{C} \mathrm{NMR}$ $\left(\mathrm{CDCl}_{3}, 75 \mathrm{MHz}\right) \delta: 194.8,171.8,135.1,129.9,129.1$, 126.6, 63.2, 49.6, 46.7, 34.0, 32.4, 31.5, 12.5, 11.6. Anal. calcd for $\mathrm{C}_{16} \mathrm{H}_{23} \mathrm{NO}_{2} \mathrm{~S}_{2}$ : C 59.04, H 7.12, N 4.30; found C 59.05, H 7.19, N 4. 31.

3-( $N$-芐氨基二硫代甲酸基)丙酸(2-苯硫基乙基)酯 $(3 g)$ : 淡黄色油状液体. ${ }^{1} \mathrm{H}$ NMR $\left(\mathrm{CDCl}_{3}, 300 \mathrm{MHz}\right) \delta$ : $7.21 \sim 7.42(\mathrm{~m}, 10 \mathrm{H}), 4.90(\mathrm{~d}, J=5.0 \mathrm{~Hz}, 2 \mathrm{H}), 4.56$ (br, $1 \mathrm{H}), 4.26(\mathrm{t}, J=6.6 \mathrm{~Hz}, 2 \mathrm{H}), 3.55$ (t, $J=6.9 \mathrm{~Hz}, 2 \mathrm{H}), 3.16$ (t, $J=6.6 \mathrm{~Hz}, 2 \mathrm{H}), 2.77(\mathrm{t}, J=6.6 \mathrm{~Hz}, 2 \mathrm{H}) ;{ }^{13} \mathrm{C}$ NMR $\left(\mathrm{CDCl}_{3}, 75 \mathrm{MHz}\right) \delta: 197.2,171.6,136.0,135.0,130.1$, 129.1, 128.9, 128.3, 128.2, 126.7, 63.3, 51.3, 34.2, 32.5, 30.2. Anal. calcd for $\mathrm{C}_{19} \mathrm{H}_{21} \mathrm{NO}_{2} \mathrm{~S}_{2}$ : C 63.48, H 5.89, N 3.90; found C 63.50, H 5.84, N 3.88.

3-(1-哌啶烷二硫代氨基甲酸基)丙酸-2-(2-萗硫基乙 基)酯(3h): 淡黄色油状液体. ${ }^{1} \mathrm{H} \mathrm{NMR}\left(\mathrm{CDCl}_{3}, 300 \mathrm{MHz}\right)$ $\delta: 7.77 \sim 7.86(\mathrm{~m}, 4 \mathrm{H}), 7.44 \sim 7.52(\mathrm{~m}, 3 \mathrm{H}), 4.33(\mathrm{t}, J=6.9$ $\mathrm{Hz}, 2 \mathrm{H}), 4.28(\mathrm{br}, 2 \mathrm{H}), 3.85(\mathrm{br}, 2 \mathrm{H}), 3.56(\mathrm{t}, J=6.6 \mathrm{~Hz}$, 2H), $3.26(\mathrm{t}, J=6.9 \mathrm{~Hz}, 2 \mathrm{H}), 2.79(\mathrm{t}, J=6.6 \mathrm{~Hz}, 2 \mathrm{H}), 1.69$ (s, $6 \mathrm{H}) ;{ }^{13} \mathrm{C} \mathrm{NMR}\left(\mathrm{CDCl}_{3}, 75 \mathrm{MHz}\right) \delta: 194.7,171.8,133.7$, $132.5,131.9,128.7,127.9,127.7,127.6,127.2,126.7$, $125.9,63.1,51.4,34.0,32.2,31.5,25.4,24.2$. Anal. calcd for $\mathrm{C}_{21} \mathrm{H}_{25} \mathrm{NO}_{2} \mathrm{~S}_{2}$ : C 65.08, $\mathrm{H}$ 6.50, N 3.61; found C 65.11, H 6.48, N 3.65.

3-( N,N-二乙氨基二硫代甲酸基)丙酸-2-(2-菜硫基乙 
基)酯(3i): 淡黄色油状液体. ${ }^{1} \mathrm{H} \mathrm{NMR}\left(\mathrm{CDCl}_{3}, 300 \mathrm{MHz}\right)$ $\delta: 7.78 \sim 7.87(\mathrm{~m}, 4 \mathrm{H}), 7.44 \sim 7.52(\mathrm{~m}, 3 \mathrm{H}), 4.33(\mathrm{t}, J=6.9$ $\mathrm{Hz}, 2 \mathrm{H}), 4.05$ (q, $J=3.2 \mathrm{~Hz}, 2 \mathrm{H}), 3.73$ (q, $J=3.4 \mathrm{~Hz}, 2 \mathrm{H})$, $3.54(\mathrm{t}, J=6.7 \mathrm{~Hz}, 2 \mathrm{H}), 3.26(\mathrm{t}, J=6.9 \mathrm{~Hz}, 2 \mathrm{H}), 2.79(\mathrm{t}$, $J=6.7 \mathrm{~Hz}, 2 \mathrm{H}), 1.27$ (t, $J=7.0 \mathrm{~Hz}, 6 \mathrm{H}) ;{ }^{13} \mathrm{C} \mathrm{NMR}\left(\mathrm{CDCl}_{3}\right.$, $75 \mathrm{MHz}) \delta$ : 194.8, 171.8, 133.7, 132.5, 132.0, 128.7, 128.0, 127.7, 127.6, 127.2, 126.7, 125.9, 63.1, 49.5, 46.7, 33.9, 32.3, 31.5, 12.5, 11.6. Anal. calcd for $\mathrm{C}_{20} \mathrm{H}_{25} \mathrm{NO}_{2} \mathrm{~S}_{2}$ : C 63.96, H 6.71, N 3.73; found C 63.97, H 6.74, N 3.76.

3-( $\mathrm{N}$-苠氨基二硫代甲酸基)丙酸-2-(2-荟硫基乙基) 酯 $(\mathbf{3 j})$ : 淡黄色油状液体. ${ }^{1} \mathrm{H} \mathrm{NMR}\left(\mathrm{CDCl}_{3}, 300 \mathrm{MHz}\right) \delta$ : $7.78 \sim 7.87(\mathrm{~m}, 4 \mathrm{H}), 7.46 \sim 7.51(\mathrm{~m}, 3 \mathrm{H}), 7.28 \sim 7.38(\mathrm{~m}$, $5 \mathrm{H}), 4.91(\mathrm{~d}, J=4.9 \mathrm{~Hz}, 1 \mathrm{H}), 4.57(\mathrm{br}, 1 \mathrm{H}), 4.33$ (t, $J=6.9$ $\mathrm{Hz}, 2 \mathrm{H}), 3.51$ (t, $J=6.6 \mathrm{~Hz}, 2 \mathrm{H}), 3.26$ (t, $J=6.9 \mathrm{~Hz}, 2 \mathrm{H})$, $2.77(\mathrm{t}, J=6.6 \mathrm{~Hz}, 2 \mathrm{H}) ;{ }^{13} \mathrm{C} \mathrm{NMR}\left(\mathrm{CDCl}_{3}, 75 \mathrm{MHz}\right) \delta$ : $197.2,171.6,136.1,133.7,132.5,132.0,128.9,128.7$, $128.6,128.3,128.2,128.0,127.7,127.1,126.6,126.0$, 63.3, 51.3, 34.2, 32.3, 30.2. Anal. calcd for $\mathrm{C}_{23} \mathrm{H}_{23} \mathrm{NO}_{2} \mathrm{~S}_{2}$ : C 67.45, H 5.66, N 3.42; found C 67.47, H 5.67, N 3.46.

1-吡咯基二硫代氨基甲酸烯丙酯(4a): 淡黄色油状 液体 ${ }^{[15]}$. ${ }^{1} \mathrm{H}$ NMR $\left(\mathrm{CDCl}_{3}, 300 \mathrm{MHz}\right) \delta: 5.87 \sim 5.96(\mathrm{~m}$, $1 \mathrm{H}), 5.25 \sim 5.31(\mathrm{dd}, J=16.8 \mathrm{~Hz}, 1.2 \mathrm{~Hz}, 1 \mathrm{H}), 5.12(\mathrm{~d}, J=$ $9.9 \mathrm{~Hz}, 1 \mathrm{H}), 4.00(\mathrm{dd}, J=6.9 \mathrm{~Hz}, 0.75 \mathrm{~Hz}, 2 \mathrm{H}), 3.91(\mathrm{t}$, $J=6.7 \mathrm{~Hz}, 2 \mathrm{H}), 3.63(\mathrm{t}, J=6.6 \mathrm{~Hz}, 2 \mathrm{H}), 1.91 \sim 2.11(\mathrm{~m}$, $4 \mathrm{H}) ;{ }^{13} \mathrm{C} \mathrm{NMR}\left(\mathrm{CDCl}_{3}, 75 \mathrm{MHz}\right) \delta: 192.1,132.9,118.3$, 55.0, 50.5, 39.5, 26.0, 24.2.

1-哌啶基二硫代氨基甲酸烯丙酯(4b): 淡黄色油状 液体 ${ }^{[15]}$. ${ }^{1} \mathrm{H}$ NMR $\left(\mathrm{CDCl}_{3}, 300 \mathrm{MHz}\right) \delta: 5.86 \sim 5.98(\mathrm{~m}$, $1 \mathrm{H}), 5.30(\mathrm{dd}, J=16.8 \mathrm{~Hz}, 1.0 \mathrm{~Hz}, 1 \mathrm{H}), 5.14$ (d, $J=9.9$ $\mathrm{Hz}, 1 \mathrm{H}), 4.27$ (br, 2H), 4.00 (d, J=6.9 Hz, 2H), 3.88 (br 2H), 1.69 (br, 6H); ${ }^{13} \mathrm{C} \mathrm{NMR}\left(\mathrm{CDCl}_{3}, 75 \mathrm{MHz}\right) \delta$ : 195.1, 132.8, 118.4, 51.4, 40.3, 25.6, 24.3.

$N, N$ 二 二乙氨基二硫代甲酸烯丙酯 $(4 \mathbf{c})$ : 淡黄色油状 液体 ${ }^{[15]} .{ }^{1} \mathrm{H}$ NMR $\left(\mathrm{CDCl}_{3}, 300 \mathrm{MHz}\right) \delta: 5.86 \sim 6.00(\mathrm{~m}$, $1 \mathrm{H}), 5.27 \sim 5.33(\mathrm{dd}, J=16.8,1.0 \mathrm{~Hz}, 1 \mathrm{H}), 5.14$ (d, $J=9.9$ $\mathrm{Hz}, 1 \mathrm{H}), 4.02$ (q, $J=3.2 \mathrm{~Hz}, 2 \mathrm{H}), 3.98$ (d, $J=6.9 \mathrm{~Hz}, 2 \mathrm{H})$, $3.74(\mathrm{q}, J=3.2 \mathrm{~Hz}, 2 \mathrm{H}), 1.27 \sim 1.30(\mathrm{~m}, 6 \mathrm{H}) ;{ }^{13} \mathrm{C} \mathrm{NMR}$ $\left(\mathrm{CDCl}_{3}, 75 \mathrm{MHz}\right) \delta: 195.0,132.7,118.4,49.5,46.7,40.3$, 12.4, 11.5 .

1-苠氨基二硫代甲酸烯丙酯(4d): 淡黄色油状液 体 ${ }^{[15]} .{ }^{1} \mathrm{H} \mathrm{NMR}\left(\mathrm{CDCl}_{3}, 300 \mathrm{MHz}\right) \delta: 7.33 \sim 7.35$ (m, 2H), $7.28 \sim 7.33(\mathrm{~m}, 3 \mathrm{H}), 7.11$ (br, $1 \mathrm{H}), 5.84 \sim 5.98(\mathrm{~m}, 1 \mathrm{H})$, $5.26(\mathrm{~d}, J=9.9 \mathrm{~Hz}, 1 \mathrm{H}), 5.15$ (d, $J=9.9 \mathrm{~Hz}, 1 \mathrm{H}), 4.90$ (d, $J=6.9 \mathrm{~Hz}, 2 \mathrm{H}), 3.94(\mathrm{~d}, J=6.6 \mathrm{~Hz}, 2 \mathrm{H}) ;{ }^{13} \mathrm{C} \mathrm{NMR}$ $\left(\mathrm{CDCl}_{3}, 75 \mathrm{MHz}\right) \delta: 195.2,137.8,131.9,128.9,126.5$,
126.4, 118.4, 51.6, 39.9.

1-吡咯基二硫代氨基甲酸炔丙酯(4e): 淡黄色油状 液体. ${ }^{1} \mathrm{H} \mathrm{NMR}\left(\mathrm{CDCl}_{3}, 300 \mathrm{MHz}\right) \delta: 4.11(\mathrm{~d}, J=2.6 \mathrm{~Hz}$, $2 \mathrm{H}), 3.93(\mathrm{t}, J=6.8 \mathrm{~Hz}, 2 \mathrm{H}), 3.64(\mathrm{t}, J=6.6 \mathrm{~Hz}, 2 \mathrm{H}), 2.23$ $(\mathrm{t}, J=2.7 \mathrm{~Hz}, 1 \mathrm{H}), 1.94 \sim 2.13(\mathrm{~m}, 4 \mathrm{H}) ;{ }^{13} \mathrm{C} \mathrm{NMR}\left(\mathrm{CDCl}_{3}\right.$, $75 \mathrm{MHz}) \delta: 190.5,78.7,71.3,55.2,50.5,26.1,25.2,24.2$. Anal. calcd for $\mathrm{C}_{8} \mathrm{H}_{11} \mathrm{NS}_{2}$ : C 51.85, H 5.98, N 7.56; found C 51.87, H 5.97, N 7.60.

1-哌啶基二硫代氨基甲酸炔丙酯(4f): 淡黄色油状 液体. ${ }^{1} \mathrm{H}$ NMR $\left(\mathrm{CDCl}_{3}, 300 \mathrm{MHz}\right) \delta: 4.26(\mathrm{br}, 2 \mathrm{H}), 4.10$ (d, $J=2.5 \mathrm{~Hz}, 2 \mathrm{H}), 3.85$ (br 2H), 2.23 (t, $J=2.5 \mathrm{~Hz}, 1 \mathrm{H})$, 1.69 (br, 6H); ${ }^{13} \mathrm{C} \mathrm{NMR}\left(\mathrm{CDCl}_{3}, 75 \mathrm{MHz}\right) \delta: 193.5,78.5$, 71.5, 51.1, 26.0, 25.7, 24.2. Anal. calcd for $\mathrm{C}_{9} \mathrm{H}_{13} \mathrm{NS}_{2}$ : C 54.23, H 6.57, N 7.03; found C 54.25, H 6.59, N 7.06.

$N, N$-二乙氨基二硫代甲酸炔丙酯 $(\mathbf{4 g})$ : 淡黄色油状 液体. ${ }^{1} \mathrm{H} \mathrm{NMR}\left(\mathrm{CDCl}_{3}, 300 \mathrm{MHz}\right) \delta: 4.08(\mathrm{~d}, J=2.7 \mathrm{~Hz}$, 2H), 4.02 (q, $J=3.1 \mathrm{~Hz}, 2 \mathrm{H}), 3.72$ (q, $J=4.6 \mathrm{~Hz}, 2 \mathrm{H}), 2.23$ $(\mathrm{t}, J=2.5 \mathrm{~Hz}, 1 \mathrm{H}), 1.22 \sim 1.32(\mathrm{~m}, 6 \mathrm{H}) ;{ }^{13} \mathrm{C} \mathrm{NMR}\left(\mathrm{CDCl}_{3}\right.$, $75 \mathrm{MHz}) \delta: 193.3,78.5,71.5,49.7,46.8,25.9,12.5,11.5$. Anal. calcd for $\mathrm{C}_{8} \mathrm{H}_{13} \mathrm{NS}_{2}$ : C 51.29, $\mathrm{H}$ 6.99, N 7.48; found C 51.26, H 6.96, N 7.02.

1 - 苠氨基二硫代甲酸炔丙酯(4h): 淡黄色油状液体. ${ }^{1} \mathrm{H}$ NMR $\left(\mathrm{CDCl}_{3}, 300 \mathrm{MHz}\right) \delta: 7.31 \sim 7.41(\mathrm{~m}, 2 \mathrm{H})$, $7.28 \sim 7.32(\mathrm{~m}, 3 \mathrm{H}), 7.11(\mathrm{br}, 1 \mathrm{H}), 4.13(\mathrm{~d}, J=2.7 \mathrm{~Hz}$, $2 \mathrm{H}), 4.09(\mathrm{~d}, J=2.7 \mathrm{~Hz}, 2 \mathrm{H}), 2.21(\mathrm{t}, J=2.5 \mathrm{~Hz}, 1 \mathrm{H}) ;{ }^{13} \mathrm{C}$ NMR $\left(\mathrm{CDCl}_{3}, 75 \mathrm{MHz}\right) \delta$ : 193.3, 137.9, 131.8, 128.6, 126.7, 78.5, 71.5, 52.8 , 25.9. Anal. calcd for $\mathrm{C}_{11} \mathrm{H}_{11} \mathrm{NS}_{2}$ : C 59.69, H 5.01, N 6.33; found C 59.66, H 5.03, N 6.32.

1-吡咯基二硫代氨基甲酸苠酯(4i): 淡黄色油状液 体 ${ }^{[15]} .{ }^{1} \mathrm{H} \mathrm{NMR}\left(\mathrm{CDCl}_{3}, 300 \mathrm{MHz}\right) \delta: 7.38 \sim 7.41(\mathrm{~m}, 2 \mathrm{H})$, $7.27 \sim 7.33(\mathrm{~m}, 3 \mathrm{H}), 4.58(\mathrm{~s}, 2 \mathrm{H}), 3.94(\mathrm{t}, J=6.7 \mathrm{~Hz}, 2 \mathrm{H})$, $3.62(\mathrm{t}, J=6.9 \mathrm{~Hz}, 2 \mathrm{H}), 1.92 \sim 2.10(\mathrm{~m}, 4 \mathrm{H}) ;{ }^{13} \mathrm{C} \mathrm{NMR}$ $\left(\mathrm{CDCl}_{3}, 75 \mathrm{MHz}\right) \delta: 192.3,136.5,129.2,128.5,127.4$, 55.0, 50.5, 41.2, 26.1, 24.3.

1-哌啶基二硫代氨基甲酸芐酯 $\mathbf{4} \mathbf{j})$ : 淡黄色油状液 体 ${ }^{[15]} .{ }^{1} \mathrm{H}$ NMR $\left(\mathrm{CDCl}_{3}, 300 \mathrm{MHz}\right) \delta: 7.38 \sim 7.41(\mathrm{~m}, 2 \mathrm{H})$, $7.27 \sim 7.34(\mathrm{~m}, 3 \mathrm{H}), 4.56(\mathrm{~s}, 2 \mathrm{H}), 4.30(\mathrm{br}, 2 \mathrm{H}), 3.86(\mathrm{br}$ 2H), 1.69 (br, 6H); ${ }^{13} \mathrm{C} \mathrm{NMR}\left(\mathrm{CDCl}_{3}, 75 \mathrm{MHz}\right) \delta: 195.3$, 136.1, 129.4, 128.6, 127.4, 52.5, 42.2, 25.8, 24.3.

$N, N$-二乙氨基二硫代甲酸苠酯 $(4 \mathbf{k})$ : 淡黄色油状液 体 ${ }^{[15]} .{ }^{1} \mathrm{H} \mathrm{NMR}\left(\mathrm{CDCl}_{3}, 300 \mathrm{MHz}\right) \delta: 7.38 \sim 7.41(\mathrm{~m}, 2 \mathrm{H})$, $7.23 \sim 7.34(\mathrm{~m}, 3 \mathrm{H}), 4.54(\mathrm{~s}, 2 \mathrm{H}), 4.05(\mathrm{q}, J=3.2 \mathrm{~Hz}, 2 \mathrm{H})$, $3.72(\mathrm{q}, J=3.6 \mathrm{~Hz}, 2 \mathrm{H}), 1.27 \sim 1.30(\mathrm{~m}, 6 \mathrm{H}) ;{ }^{13} \mathrm{C} \mathrm{NMR}$ $\left(\mathrm{CDCl}_{3}, 75 \mathrm{MHz}\right) \delta: 195.3,136.0,129.3,128.5,127.4$, 49.4, 46.7, 42.2, 12.5, 11.6. 
1-芐氨基二硫代甲酸苠酯(4I): 淡黄色油状液体 ${ }^{[15]}$. ${ }^{1} \mathrm{H}$ NMR $\left(\mathrm{CDCl}_{3}, 300 \mathrm{MHz}\right) \delta: 7.33 \sim 7.46(\mathrm{~m}, 4 \mathrm{H})$, $7.13 \sim 7.31(\mathrm{~m}, 6 \mathrm{H}), 6.41(\mathrm{br}, 1 \mathrm{H}), 4.78(\mathrm{~d}, J=6.9 \mathrm{~Hz}$, 2H), $4.60(\mathrm{~s}, 2 \mathrm{H}) ;{ }^{13} \mathrm{C} \mathrm{NMR}\left(\mathrm{CDCl}_{3}, 75 \mathrm{MHz}\right) \delta$ : 197.3, $136.0,132.7,128.9,128.3,128.2,127.9,126.9,118.5$, $51.2,38.5$.

1-哌啶基二硫代氨基甲酸-2-(2-噻吩乙基)酯(4m): 淡黄色油状液体. ${ }^{1} \mathrm{H}$ NMR $\left(\mathrm{CDCl}_{3}, 300 \mathrm{MHz}\right) \delta: 7.13 \sim$ $7.15(\mathrm{dd}, J=5.0,1.0 \mathrm{~Hz}, 1 \mathrm{H}), 6.88 \sim 6.95(\mathrm{~m}, 2 \mathrm{H}), 4.29$ (br, 2H), 3.89 (br, 2H), 3.60 (t, $J=6.5 \mathrm{~Hz}, 2 \mathrm{H}), 3.23$ (t, $J=$ $6.5 \mathrm{~Hz}, 2 \mathrm{H}), 1.69$ (brs, 6H); ${ }^{13} \mathrm{C} \mathrm{NMR}\left(\mathrm{CDCl}_{3}, 75 \mathrm{MHz}\right) \delta$ : 195.1, 142.8, 126.8, 125.1, 123.6, 52.2, 38.2, 29.6, 26.5, 24.3. Anal. calcd for $\mathrm{C}_{12} \mathrm{H}_{17} \mathrm{NS}_{3}$ : C 53.09, H 6.31, N 5.16; found C 53.11, H 6.34, N 5. 14.

$N, N$-二乙氨基二硫代甲酸-2-(2-噻吩乙基)酯(4n): 淡黄色油状液体. ${ }^{1} \mathrm{H}$ NMR $\left(\mathrm{CDCl}_{3}, 300 \mathrm{MHz}\right) \delta: 7.13 \sim$ $7.15(\mathrm{dd}, J=5.0,1.0 \mathrm{~Hz}, 1 \mathrm{H}), 6.88 \sim 6.95(\mathrm{~m}, 2 \mathrm{H}), 4.04$ (q, $J=3.5 \mathrm{~Hz}, 2 \mathrm{H}), 3.73(\mathrm{q}, J=3.6 \mathrm{~Hz}, 2 \mathrm{H}), 3.58$ (t, $J=6.5$ $\mathrm{Hz}, 2 \mathrm{H}), 3.23$ (t, $J=6.5 \mathrm{~Hz}, 2 \mathrm{H}), 1.27$ (t, $J=6.5 \mathrm{~Hz}, 6 \mathrm{H})$; ${ }^{13} \mathrm{C}$ NMR $\left(\mathrm{CDCl}_{3}, 75 \mathrm{MHz}\right) \delta: 195.1,142.8,126.8,125.1$, 123.6, 49.5, 46.7, 38.2, 29.5, 12.5, 11.6. Anal. calcd for $\mathrm{C}_{11} \mathrm{H}_{17} \mathrm{NS}_{3}$ : C 50.92, H 6.60, N 5.40; found $\mathrm{C} 50.90, \mathrm{H}$ 6.62, N 5. 44.

1-苠氨基二硫代甲酸-2-(2-噻吩乙基)酯(4o): 淡黄 色固体, m.p. 55 56 ${ }^{\circ} \mathrm{C}^{[15]}$. ${ }^{1} \mathrm{H}$ NMR $\left(\mathrm{CDCl}_{3}, 300 \mathrm{MHz}\right)$ $\delta: 7.33 \sim 7.35(\mathrm{~m}, 5 \mathrm{H}), 7.15(\mathrm{~d}, J=5.1 \mathrm{~Hz}, 1 \mathrm{H}), 7.08(\mathrm{br}$, $1 \mathrm{H}), 6.78 \sim 6.95(\mathrm{~m}, 2 \mathrm{H}), 4.90(\mathrm{~d}, J=4.8 \mathrm{~Hz}, 2 \mathrm{H}), 3.60(\mathrm{t}$, $J=6.5 \mathrm{~Hz}, 2 \mathrm{H}), 3.23(\mathrm{t}, J=6.5 \mathrm{~Hz}, 2 \mathrm{H}) ;{ }^{13} \mathrm{C} \mathrm{NMR}\left(\mathrm{CDCl}_{3}\right.$, $75 \mathrm{MHz}) \delta$ : 197.5, 142.4, 136.1, 128.9, 128.3, 128.1, 126.9, 125.2, 123.8, 51.3, 36.9, 29.8. Anal. calcd for $\mathrm{C}_{14} \mathrm{H}_{15} \mathrm{NS}_{3}$ : C 57.30, H 5.15, N 4.77; found C 57.31, H 5.14, N, 4. 76.

1-丁胺基二硫代甲酸-2-(2-噻吩乙基)酯(4p): 淡黄 色油状液体. ${ }^{1} \mathrm{H}$ NMR $\left(\mathrm{CDCl}_{3}, 300 \mathrm{MHz}\right) \delta: 7.15(\mathrm{~d}, J=$ $4.9 \mathrm{~Hz}, 1 \mathrm{H}), 6.88 \sim 6.95(\mathrm{~m}, 2 \mathrm{H}), 3.71$ (q, $J=3.8 \mathrm{~Hz} 2 \mathrm{H})$, $3.54(\mathrm{t}, J=6.5 \mathrm{~Hz}, 2 \mathrm{H}), 3.42(\mathrm{br}, 1 \mathrm{H}), 3.23(\mathrm{t}, J=6.5 \mathrm{~Hz}$, $2 \mathrm{H}), 1.57 \sim 1.67(\mathrm{~m}, 2 \mathrm{H}), 1.35 \sim 1.45(\mathrm{~m}, 2 \mathrm{H}), 0.94(\mathrm{t}, J=$ $7.1 \mathrm{~Hz}, 2 \mathrm{H}) ;{ }^{13} \mathrm{C} \mathrm{NMR}\left(\mathrm{CDCl}_{3}, 75 \mathrm{MHz}\right) \delta: 197.2,142.5$, 126.8, 125.2, 123.7, 47.0, 36.7, 30.4, 29.9, 20.1, 13.7. Anal. calcd for $\mathrm{C}_{11} \mathrm{H}_{17} \mathrm{NS}_{3}$ : C 50.92, H 6.60, N 5.40; found C 50.96, H 6.66, N 5. 42.

\section{2 结果与讨论}

\section{1 溶剂及温度对反应的影响}

首先以苯基乙烯基砜、四氢吡咯、 $\mathrm{CS}_{2}$ 的反应为模
板, 研究溶剂对反应的影响(表 1). 利用常用的溶剂如 $\mathrm{DMF}$ 、水、乙醇、氯仿、乙腈及 $\mathrm{THF}$ 等为反应溶剂，按 第 1.2 节所述的实验步骤进行实验，反应 $2 \mathrm{~h}$ 后，反应收 率结果见表 1 .

表 1 反应溶剂对反应产率的影响

Table 1 The effect of solvents on the yield
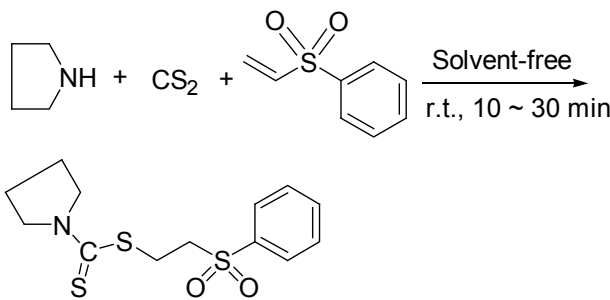

\begin{tabular}{cccccccc}
\hline Solvent & DMF & $\mathrm{H}_{2} \mathrm{O}$ & $\mathrm{CH}_{3} \mathrm{CH}_{2} \mathrm{OH} \mathrm{CH}_{3} \mathrm{Cl}_{\mathrm{CH}_{3} \mathrm{CN}}$ & $\mathrm{THF}$ & Neat \\
\hline Yield/\% & 80 & 83 & 85 & 79 & 88 & 80 & 96 \\
\hline
\end{tabular}

从上述实验结果可以看出, 该反应可以在大多数溶 剂中反应，有中等偏上的产率，在无溶剂条件下，可以 得到最佳的收率. 但值得注意的是在无溶剂的条件下， 反应起始阶段要在冰浴下反应，在常温下脂肪胺与 $\mathrm{CS}_{2}$ 反应非常迅速，容易得到硫嫝类化合物，同时控制脂肪 胺的滴加速度也极为重要.

\section{2 反应可能的机理与反应原料的物质的量比探索}

从相关文献中我们可以看出该反应实际上是一个 Michael 加成反应, 由此我们推断出可能反应机理为如 Scheme 2 所示，由于脂肪胺与 $\mathrm{CS}_{2}$ 可以迅速的反应，因 而首先应该是生成脂肪胺与 $\mathrm{CS}_{2}$ 反应的中间体二硫代甲 酸，随后颈基对活化的 $\alpha, \beta$-不饱和化合物进行 Michael 加成反应.

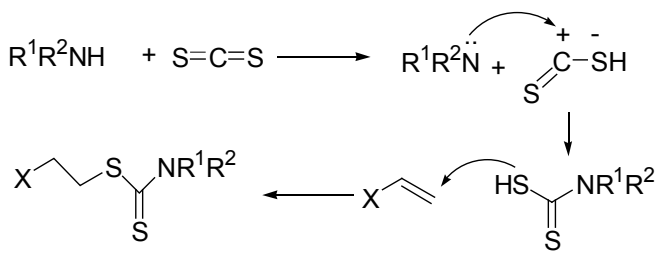

Scheme 2

根据上述反应的可能机理，我们对反应各原料之间 的物质的量比进行了适当的优化, 实验发现脂肪胺、 $\mathrm{CS}_{2}$ 与 $\alpha, \beta$-不饱和化合物或卤代烃之间物质的量比为 $1.125: 1.5: 1$ 可得最佳的反应产率, 脂肪胺与 $\mathrm{CS}_{2}$ 的物 质的量比为 $1: 1$ 时, 反应的产率只有 $78 \%$, 但加大 $\mathrm{CS}_{2}$ 的物质的量比, 如脂肪胺与 $\mathrm{CS}_{2}$ 的物质的量比为 $2: 1$ 或是 $3: 1$ 时, 反应产率与物质的量比为 $1.5: 1$ 时相比, 未见产率明显升高，产率在 $96 \%$ 左右. 


\section{3 无溶剂条件下 $\alpha, \beta$-不饱和化合物、胺和 $\mathrm{CS}_{2}$ 的反 应}

利用上述优化好的反应条件, 即在无溶剂无催化 剂, 反应底物脂肪胺、 $\mathrm{CS}_{2}$ 与 $\alpha, \beta$-不饱和化合物或卤代 烃之间物质的量比为 $1.125: 1.5: 1$ 的情况下进行反应, $\mathrm{CS}_{2}$ 与 $\alpha, \beta$-不饱和化合物的混合物冰浴冷却到 $0{ }^{\circ} \mathrm{C}$ 后滴 入脂肪胺, 再在室温下搅拌 $30 \mathrm{~min} \sim 2 \mathrm{~h}$, 反应就可较好 地完成(Eq. 1, 表 2). 从实验结果可以看出脂肪胺如伯 胺或仲胺可以顺利地反应, 芳胺如对甲氧基苯胺未能观

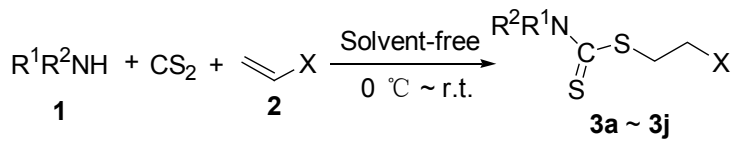

察到有丙烯酸酯可以较好地参与反应，高产率地生成目 标化合物, 但未活化的 $\alpha, \beta$-不饱和化合物如苯基乙烯基 硫醚或苯基乙烯基醚未能参与反应.

\section{4 无溶剂条件下卤代烃、胺和 $\mathrm{CS}_{2}$ 的反应}

利用上述的反应条件, 把 $\alpha, \beta$-不饱和化合物换成卤 代烃时, 反应同样可以得到二硫代氨基甲酸酯产物(Eq. 2), 实验结果见表 3. 实验发现芐溴、烯丙基澳、炔丙基 澳及对甲苯磺酸酯均可参与该三元反应，反应的选择性 较好, 未发现胺直接与芐溴、烯丙基溴、炔丙基溴及对 甲苯磺酸酯发生取代反应的副产物, 反应产率较好, 达 到 $85 \%$ 以上，同样，由于芳香胺及氯代烃的活性较弱， 不能参与该三元反应.

表 2 无溶剂条件下一锅法合成二硫代氨基甲酸酯

Table 2 One-pot synthesis of dithiocarbamates under solvent-free conditions

\begin{tabular}{|c|c|c|c|c|}
\hline Entry & Michael acceptor & Amine $\left(\mathrm{R}^{1} \mathrm{R}^{2} \mathrm{NH}\right)$ & Product & Yield/\% \\
\hline 1 & & & $3 a$ & 96 \\
\hline 2 & & & $3 b$ & 96 \\
\hline 3 & & & $3 g$ & 95 \\
\hline 4 & & $\mathrm{NH}_{2}$ & $3 d$ & 92 \\
\hline 5 & & & $3 e$ & 95 \\
\hline 6 & & & $3 f$ & 95 \\
\hline 7 & & & $3 \mathrm{~g}$ & 90 \\
\hline
\end{tabular}




Entry Michael acceptor $\quad$ Amine $\left(\mathrm{R}^{1} \mathrm{R}^{2} \mathrm{NH}\right)$

表 3 无溶剂条件下一锅法合成 $S$-烷基二硫代氨基甲酸酯

Table 3 One-pot synthesis of $S$-alkyl dithiocarbamates under solvent-free conditions

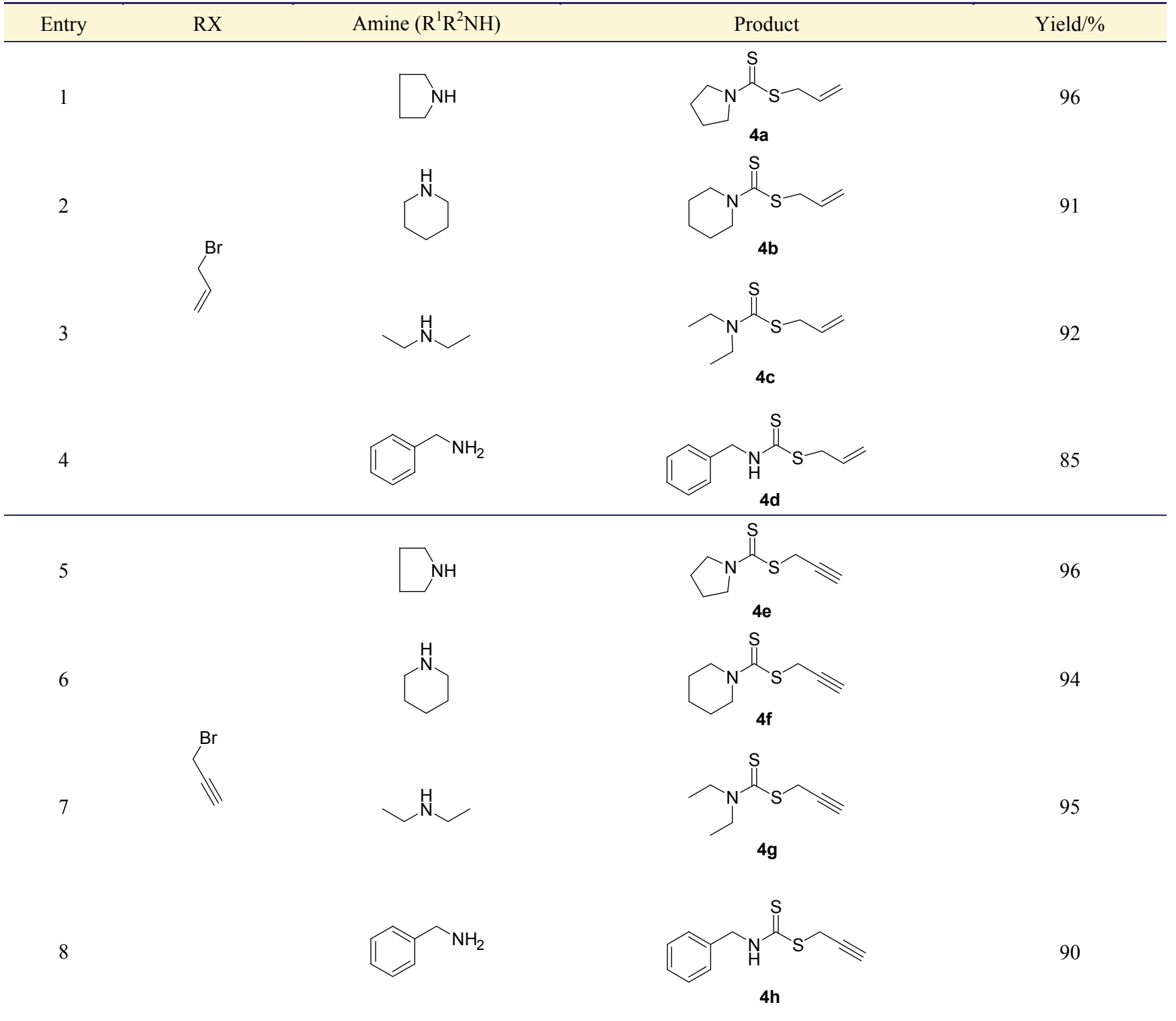




\begin{tabular}{|c|c|c|c|c|}
\hline Entry & $\mathrm{RX}$ & Amine $\left(\mathrm{R}^{1} \mathrm{R}^{2} \mathrm{NH}\right)$ & Product & Yield/\% \\
\hline 9 & & & $4 i$ & 95 \\
\hline 10 & & & $4 j$ & 91 \\
\hline 11 & & & $4 k$ & 90 \\
\hline 12 & & & 41 & 86 \\
\hline 13 & & & $4 m$ & 91 \\
\hline 14 & & & $4 n$ & 86 \\
\hline 15 & & & 40 & 88 \\
\hline 16 & & $\mathrm{NH}_{2}$ & & 90 \\
\hline
\end{tabular}

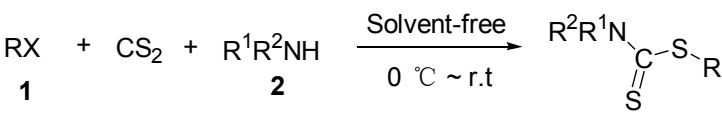

$$
\begin{aligned}
& \mathrm{X}=\mathrm{Br} \text {, OTs } \\
& 4 a \sim 4 p
\end{aligned}
$$

综上所述, 在无溶剂无催化剂的条件下, 实现了 “一锅法” 由脂肪胺、 $\mathrm{CS}_{2}$ 及 $\alpha, \beta$-不饱和化合物或卤代 烃来合成二硫代氨基甲酸酯衍生物, 该方法具有原料易 得、操作简便、反应条件温、产率较高、化学选择性较 好、可以进行工业化生产等特点, 是合成二硫代氨基甲 酸酯衍生物一种较为简便的方法.

\section{References}

[1] Boas, U.; Gertz, H.; Christensen, J. B.; Heegaard, P. M. H. Tetrahedron Lett. 2004, 45, 269.

[2] Dhooghe, M.; De Kime, N. Tetrahedron 2006, 62, 513.

[3] Suh, Y.-G.; Lee, Y.-S.; Min, K.-H.; Park, O.-H.; Kim, J.-K.; Seung, H.-S.; Seo, S.-Y.; Lee, B.-Y.; Nam, Y.-H.; Lee, K.-O.; Kim, H.-D.; Park, H.-G.; Lee, J.; Oh, U.; Lim, J.-O.; Kang, S.-U.; Kil, M.-J.; Koo, J.-y.; Shin, S. S.; Joo, Y.-H.; Kim, J. K.; Jeong, Y.-S.; Kim, S.-Y.; Park, Y.-H. J. Med. Chem. 2005, 48, 5823.

[4] Fernandez, J. M. G.; Mellet, C. O.; Blanco, J. L. J.; Mota, J. F.; Gadelle, A.; Coste-Sarguet, A.; Defaye, J. Carbohydr. Res. 1995, $268,57$.

[5] Len, C.; Boulogne-Merlot, A. S.; Postel, D.; Ronco, G.; Villa, P.; Goubert, C.; Jeufrault, E.; Mathon B.; Simon, H. J. Agric. Food Chem. 1996, 44, 2856. 
[6] Dhooghe, M.; De Kimpe, N. Tetrahedron 2006, 62, 513.

[7] Nieuwenhuizen, P. J.; Ehlers, A. W.; Haasnoot, J. G.; Janse, S. R.; Reedi, J.; Baerends, E. J. J. Am. Chem. Soc. 1999, 121, 163.

[8] Wood, M. R.; Duncalf, D. J.; Rannard, S. P.; Perrier, S. Org. Lett. 2006, 8,553 .

[9] Chin-Hsien, W. Synthesis 1981, 622.

[10] Azizi, N.; Aryanasab, F.; Torkiyan, L.; Ziyaei, A.; Saidi, M. R. J. Org. Chem. 2006, 71, 3634.
[11] Ranu, B. C.; Saha, A.; BanerJee, S. Eur. J. Org. Chem. 2008, 519.

[12] Sikchi, S. A.; Hultin, P. G. J. Org. Chem. 2006, 71, 5888.

[13] Tanaka, K.; Toda, F. Chem. Rev. 2000, 100, 1025.

[14] Yu, F.-H.; Yan, S.-J.; Lin, J. Chin. J. Org. Chem. 2010, 30, 1421 (in Chinese).

(余富朝, 严胜骄, 林军, 有机化学, 2010, 30, 1421.)

[15] Azizi, N.; Aryanasab, F.; Saidi, M. R. Org. Lett. 2006, 8, 5275.

(Zhao, X.; Lu, Z.) 